\title{
Dual Frequency Output Quasi-Resonant Inverter for Induction Heating
}

\author{
Member Shizumasa Okudaira (Tokyo Metropolitan College of Aeronautical Engineering) \\ Member Kouki Matsuse (Meiji University)
}

Keywords : quasi-resonant inverter, dual frequency, induction heating, adjustable frequency

\begin{abstract}
When such an uneven work as a gear is hardened by induction heating, it is known that concave portions of the work are heated at a lower frequency and convex portions are heated at a higher frequency which is almost three times as much as the lower frequency. It is desirable that the work is heated by both frequencies at the same time but it is impossible for a conventional resonant inverter. Then we propose a dual frequency output quasi-resonant inverter which positively generates higher harmonics. The proposed inverter has a feature that the 1st order (fundamental) current and the $3 r d$ order current are controlled by manipulating the shorting time of the resonant capacitor. The present inverter is applicable to dual frequency induction heating.
\end{abstract}

\section{Purpose and feature of this study}

The purpose of this study is to propose a novel quasi-resonant inverter which can be applied to dual frequency induction heating.

When such an uneven work as a gear is hardened by induction heating, it is known that convex portions of the work are heated at a higher frequency $f_{H}$ and concave portions of the work are heated at a lower frequency $f_{L}$. It is known that the ratio $f_{H}$ to $f_{L}$ nearly equals $3.3[1]$. That is

$$
f_{H} / f_{L} \approx 3.3
$$

It is impossible for a conventional resonant inverter to supply current for a load at both frequencies $f_{H}$ and $f_{L}$ at the same time because the output frequency depends on the resonant frequency of the load circuit [2],[3]. In order to solve the problem, we designed a new quasi-resonant inverter whose output frequency was adjustable and we reported a control method of the frequency and the power [4]-[6]. The reported inverter has two series resonant capacitors and the equivalent resonant frequency was variable by shorting one resonant capacitor. As a result, it became possible to supply two currents for the induction heater alternately, whose frequencies were adjusted to $f_{H}$ and $f_{L}$ in (1). However, it is the best way for simultaneous dual frequency induction heating that the two currents are supplied at the same time.

Then we propose a novel dual frequency output quasi-resonant inverter by improving the reported inverter, which positively generates higher harmonic currents although a conventional resonant inverter does not generate higher harmonics. The proposed inverter has a feature that the 1st order (fundamental) current and the 3rd order current are controlled by manipulating the shorting time of the resonant capacitor. And the fundamental frequency is adjustable. Equation (1) is approximately satisfied on condition that the absolute value of the 3 rd order current is made nearly equal to the absolute value of the fundamental current. Although the ratio $f_{H}$ to $f_{L}$ does not equal exactly 3.3 , the relative error of the skin depth is under a few percents. Because the skin depth is inversely proportional to a square root of the frequency. Besides, actual heated depth depends on a material and a shape of a work, and the heating time. Thus the error of the frequency is not a serious problem. According to the present inverter, it is possible to heat at two frequencies at the same time.

\section{Circuit configuration and operation principle}

Fig. 1 shows a proposed inverter circuit using Power MOSFET's as the switching devices because each on-state resistance is small. In Fig.1, $R$ represents equivalent resistance of an induction heating circuit including an iron-core matching transformer and $L$ means equivalent resistance of the circuit. This inverter consists of a current generating cir- 


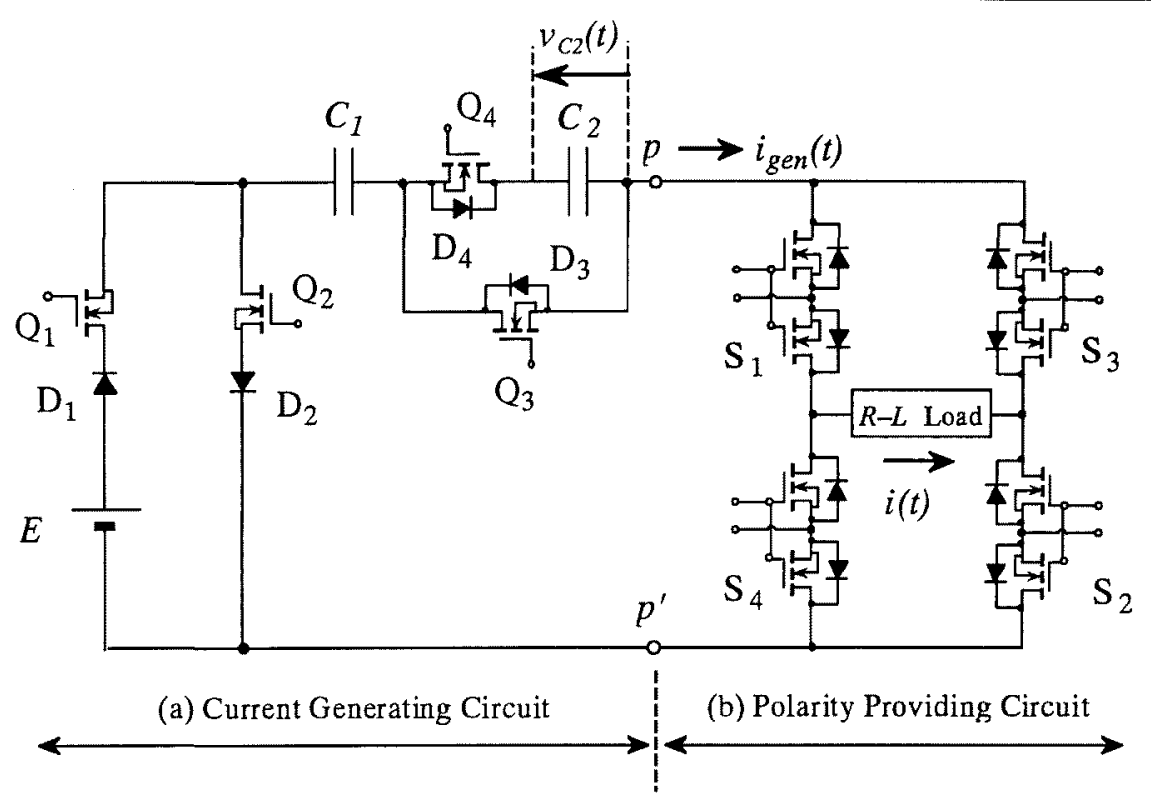

Fig.1. Proposed inverter circuit.

cuit (a) and a polarity providing circuit (b). The output terminals $p$ - $p$ ' of the circuit (a) are connected to the $R-L$ load through the direction switches $\mathrm{S}_{1}-\mathrm{S}_{4}$. The circuit (a) corresponds to the previously reported quasi-resonant inverter [4]-[6]. $C_{1}$ is the first resonant capacitor which is determined by the lowest operation frequency and $C_{2}$ is the second resonant capacitor which is determined by the highest operation frequency [4],[5], where $C_{1}$ » $C_{2}$. Each of $\mathrm{S}_{1}-\mathrm{S}_{4}$ is a two-way switch which provides the direction of the output current $i$. The body diodes of MOSFET's are used for the reverse-blocking diodes $\mathrm{D}_{3}, \mathrm{D}_{4}$ and the antiparallel diodes of the direction switches $\mathrm{S}_{1}-\mathrm{S}_{4}$.

Fig. 2 shows typical operation waveforms of the generating current $i_{g e n}$, the output current $i$ and the second capacitor voltage $v_{C 2}$, and modes classifications. The operation of the circuit (a) in each mode is disclosed as follows on condition that $Q_{1}-Q_{4}, D_{1}-D_{4}$ and $S_{1}-S_{4}$ are ideal switching devices, provided that $S_{1}-S_{4}$ are made on.

1) mode (1): The initial current is supplied under $L C_{1}$ resonant state with $\mathrm{Q}_{1}, \mathrm{Q}_{3}$ on.

2) mode (2) : The capacitor $C_{2}$ is charged under $L C_{2}$ resonant state, because of $C_{2}$ « $C_{1}$, with $\mathrm{Q}_{3}$ off and then $\mathrm{D}_{4}$ on. When the generating current $i_{\text {gen }}$ becomes zero, the diode $\mathrm{D}_{1}$, $\mathrm{D}_{4}$ turns off.

3) mode (3): The generating current $i_{g e n}$ is blocked by the diode $D_{1}, D_{4}$. The power source is separated from the circuit by the switch $Q_{1}$ off.

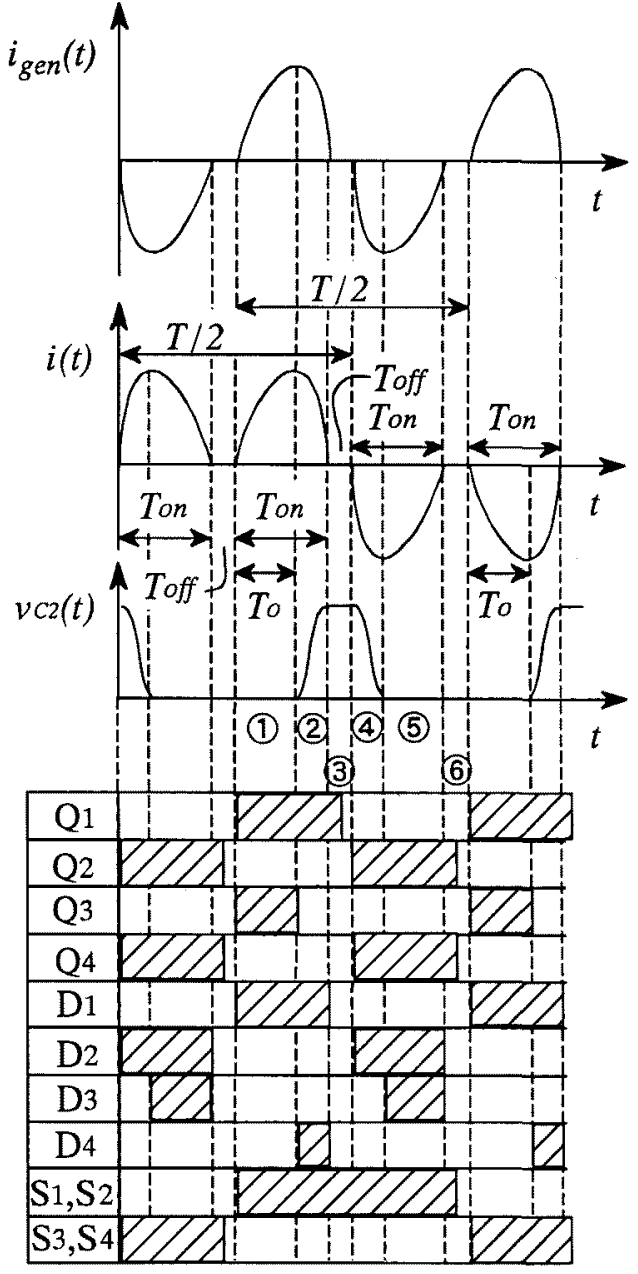

Fig.2. Typical operation waveforms and modes classifications.

T. IEE Japan, Vol. 121-D, No. 5, 2001 


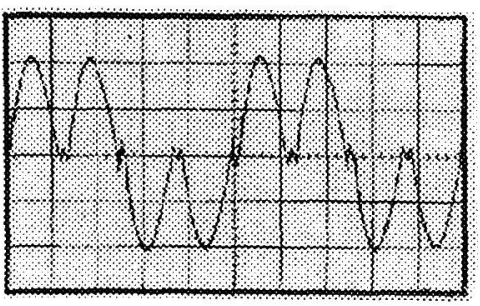

(a) $f=10 \mathrm{kHz}$

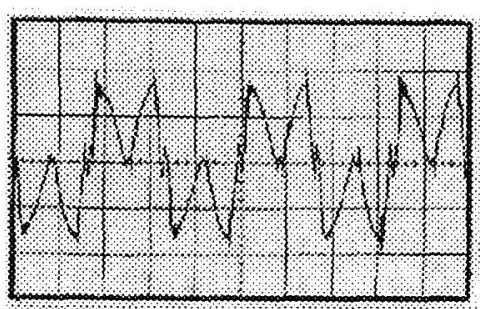

(b) $f=15 \mathrm{kHz}$

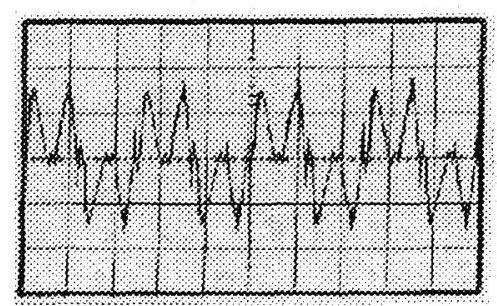

(c) $f=20 \mathrm{kHz}$

(Horizontal axis : $20 \mu \mathrm{s} / \mathrm{div}$, Vertical axis : $5 \mathrm{~A} / \mathrm{div}$ )

Fig.3. Experimental waveforms of output current $i$.

Table 1. Parameters.

\begin{tabular}{l|l}
\hline Equivalent resistance $R$ & $1.9 \Omega$ \\
\hline Equivalent inductance $L$ & $46 \mu \mathrm{H}$ \\
\hline 1st resonant capacitance $C_{1}$ & $1.0 \mu \mathrm{F}$ \\
\hline 2nd resonant capacitance $C_{2}$ & $0.1 \mu \mathrm{F}$ \\
\hline
\end{tabular}

4) mode (4): The capacitor $C_{2}$ is discharged and the reverse current $i_{\text {gen }}$ flows under $L C_{2}$ resonant state with $Q_{2}, Q_{4}$ on. When the voltage $v_{C 2}$ of capacitor $C_{2}$ returns to zero, the diode $\mathrm{D}_{3}$ turns on.

5) mode (5): The reverse current $\boldsymbol{i}_{\text {gen }}$ is decreasing under $L C_{1}$ resonant state with $\mathrm{D}_{3}$ on automatically. When the current $i_{\text {gen }}$ becomes zero, the diode $\mathrm{D}_{2}, \mathrm{D}_{3}$ turn off.

6) mode (6) : The circuit is in a state of quiescence until the beginning of the next cycle.

The output current $i$ which flows through the load is obtained, provided that $S_{1}-S_{2}$ and $S_{3}-S_{4}$ are properly actuated such as shown in Fig.2. The fundamental frequency is adjustable by changing the period $T$ and also the relative harmonic content is adjustable by changing the second capacitor shorting time $T_{0}$.

\section{Experimental waveforms}

A prototype inverter system is manufactured and the parameters are shown in Table 1 . In this circuit, the numbers of the series switching devices in every operation mode are many, that is 6 , then MOSFET is useful because its on-state resistance is low. Each MOSFET used in this inverter is 2SK1522 (Hitachi). The ratings and the electrical characteristics of it are listed in Table 2.
Table 2. Ratings and Electrical characteristics of MOSFET (2SK1522).

\begin{tabular}{l|c}
\hline Drain-to-source voltage $V_{D S}$ & $400 \mathrm{~V}$ \\
\hline Gate-to-source voltage $V_{G S}$ & $3 \mathrm{~V}$ \\
\hline Drain current $I_{D}$ & $25 \mathrm{~A}$ \\
\hline On-state resistance $R_{D S(O N)}$ & $0.11 \Omega$ \\
\hline Gate-to-source capacitance $C_{\text {iss }}$ & $8700 \mathrm{pF}$ \\
\hline Turn-on time $t_{O N}$ & $335 \mathrm{~ns}$ \\
\hline Turn-off time $t_{\text {OFF }}$ & $850 \mathrm{~ns}$ \\
\hline
\end{tabular}

Examples of the waveforms of the output current $i$ are illustrated in Fig. 3 when the d.c. power voltage $E=50 \mathrm{~V}$, the off time $T_{\text {off }}=4 \mu$ s and the operation frequency $f=10-20$ $\mathrm{kHz}$. As shown in Fig.3, it is clear that the prototype inverter operates in accordance with the theory in Fig. 2 and the operation frequency is adjustable. The higher the operation frequency is, the smaller the absolute value of the output current $i$ is because the equivalent resonant capacitance becomes smaller.

\section{Harmonics characteristics}

\subsection{Analytical results}

The relative harmonic content of the output current $\lambda_{n}$ is defined as (2).

$$
\lambda_{n}=\frac{I_{n}}{I} \quad(n=1,3,5,7, \cdots)
$$

where $I_{n}$ is the effective value of the $n$-th order output current and $I$ is the effective value of the output current. When $n=1$, 


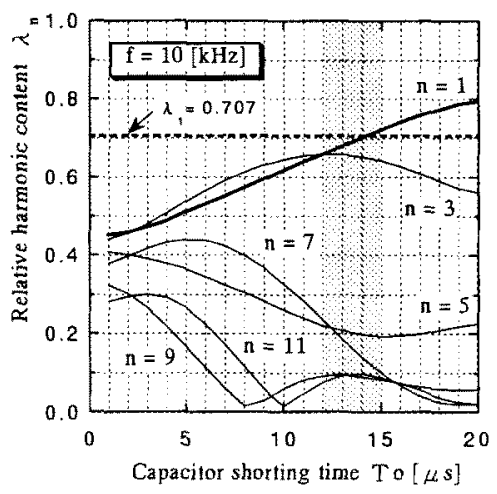

(a) $f=10 \mathrm{kHz}$

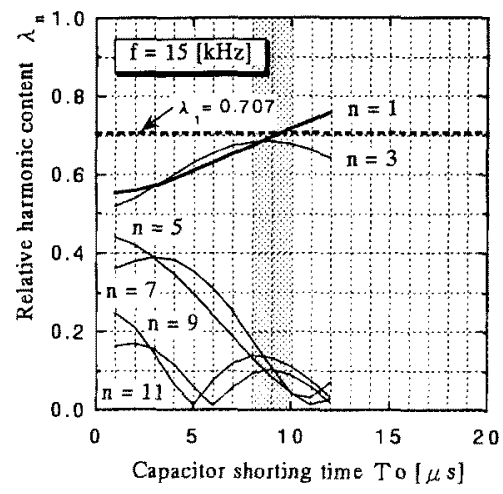

(b) $f=15 \mathrm{kHz}$

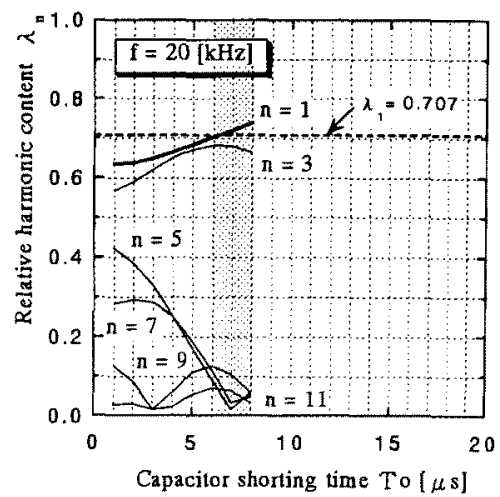

(c) $f=20 \mathrm{kHz}$

Fig.4. Analytical harmonics characteristics of output current.

$I_{1}$ means the effective value of the fundamental output current. The relationship between the harmonic current $I_{n}$ and the effective current $I$ is given by

$$
I_{1}{ }^{2}+I_{3}{ }^{2}+I_{5}{ }^{2}+I_{7}{ }^{2}+\cdots \cdot=I^{2} .
$$

Thus the sum of the square of each relative harmonic content is obtained by

$$
\lambda_{1}^{2}+\lambda_{3}^{2}+\lambda_{5}^{2}+\lambda_{7}^{2}+\cdots=1 .
$$

$\lambda_{n}{ }^{2}$ means the relative harmonic content of the electric power in (4).

Fig. 4 shows the analytical harmonics characteristics of the output current which is calculated using the parameters on Table 1 after solving the circuit equations every mode in Fig. 2 and obtaining the output current $i$. It is clear that each of the relative harmonic contents $\lambda_{n}$ depends on the resonant capacitor shorting time $T_{0}$. Further, the painted areas in Fig.4 disclose that the relative harmonic contents of more than 5 th order are smaller than the 3 rd order content when the fundamental content almost equals to the 3 rd order one. That is

$$
\lambda_{5}, \lambda_{7}, \cdots<\lambda_{3} \text {, where } \lambda_{1} \approx \lambda_{3} \text {. }
$$

In terms of power,

$$
\lambda_{5}{ }^{2}, \lambda_{7}{ }^{2}, \cdots \ll \lambda_{3}{ }^{2} \text {, where } \lambda_{1} \approx \lambda_{3} \text {. }
$$

Then the higher harmonics of more than 5th order are neglected on condition that the resonant capacitor shorting time $T_{0}$ is such manipulated as $\lambda_{1} \approx \lambda_{3}$. More over it is considered that the output current hardly contains higher harmonics except the 3 rd order harmonic wave at the higher operation frequency.

\subsection{Experimental results}

Fig.5 shows the experimental harmonics characteristics of the output current. The experimental characteristics in Fig.5 are very similar to the analytical characteristics in Fig.4. In Fig.5, it is obvious that each of the relative harmonic contents $\lambda_{n}$ is adjustable by changing the capacitor shorting time $T_{0}$. Further, in the painted areas in Fig.5, the relative harmonic contents of more than 5 th order are smaller than the 3 rd order content when the fundamental content almost equals to the 3 rd order one.

\section{Harmonics control}

The absolute values of the fundamental current and the 3 rd order current are controlled using the analytical results because it is considered that the analytical results in Fig.4 represents the experimental results in Fig.5.

In the painted areas in Fig.4, the 3rd order current about equals to the fundamental current and the relative harmonic contents of more than 5 th order can be neglected. Then equations (7) and (8) exist.

$$
\begin{aligned}
& \lambda_{3}{ }^{2}+\lambda_{5}{ }^{2}+\lambda_{7}{ }^{2}+\cdots \approx \lambda_{3}{ }^{2} \\
& \lambda_{3} \approx \lambda_{1}
\end{aligned}
$$

Equation (4) therefore becomes as

$$
\lambda_{1}^{2} \approx \frac{1}{2} \text {. }
$$

Namely

$$
\lambda_{1} \approx \frac{1}{\sqrt{2}}=0.707
$$




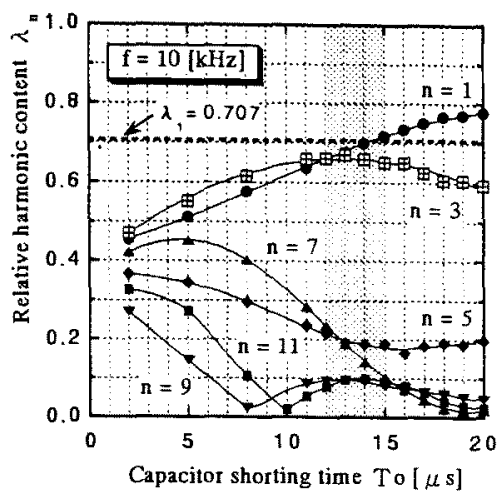

(a) $f=10 \mathrm{kHz}$

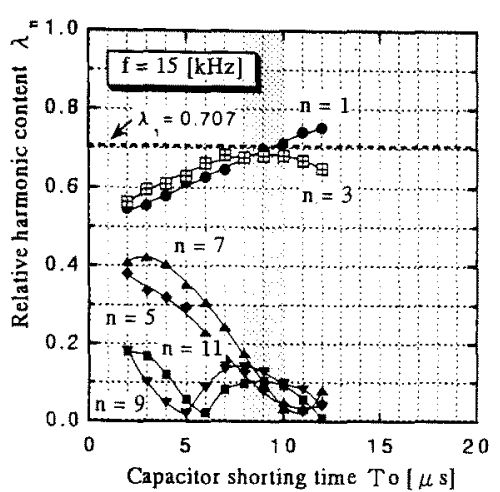

(b) $f=15 \mathrm{kHz}$

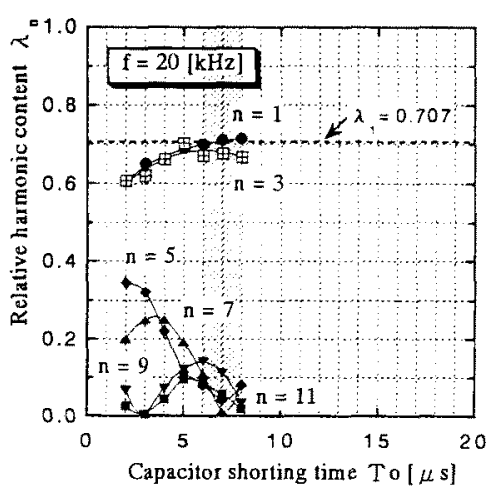

(c) $f=20 \mathrm{kHz}$

Fig.5 Experimental harmonics characteristics of output current.

Accordingly the 3 rd order current $I_{3}$ which almost equals to the fundamental current $I_{l}$ can be supplied to the load if $\lambda_{1}$ is controlled as (10) by manipulating the second resonant capacitor shorting time $T_{0}$, where their frequencies $f_{1}, f_{3}$ practically satisfy (1). At that time the higher harmonic power equals to the fundamental power.

The resonant capacitor shorting time $T_{0}{ }^{*}$ giving $\lambda_{1}=0.707$ can be obtained in every operation frequency $f$ by the analytical results illustrated in Fig. 4 . The relation between $f$ and $T_{0}{ }^{*}$ is shown in Fig.6. According to regression analysis, an approximate equation of the characteristic curve in Fig. 6 is obtained as

$$
T_{0}^{*}=33.3-2.87 f+0.120 f^{2}-0.00221 f^{3}
$$

where the unit of $f$ is $\mathrm{kHz}$ and the unit of $T_{0}^{*}$ is $\mu \mathrm{s}$. The manipulated variable $T_{0}{ }^{*}$ for the harmonics control is calculated by (11).

Experimental results of the harmonics control using (11)

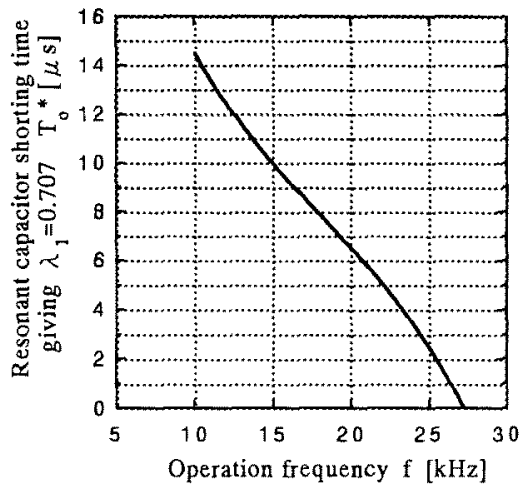

Fig. 6 Relation between the operation frequency $f$ and the capacitor shorting time $T_{0}{ }^{*}$ giving $\lambda_{1}=0.707$.

are disclosed in Fig.7. According to Fig.7, it is apparent that the fundamental current and the 3rd order current are such controlled that their values become nearly equal in every desirable operation frequency. Although the output current

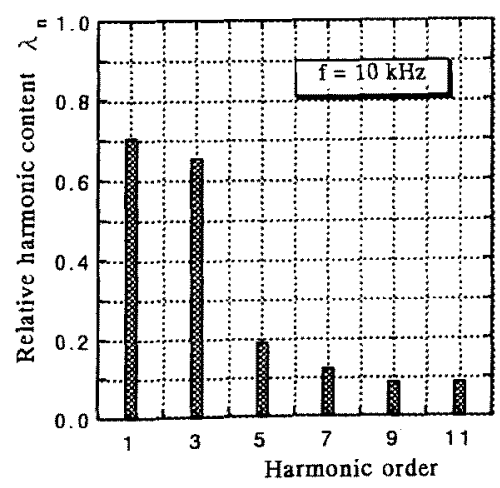

(a) $f=10 \mathrm{kHz}$

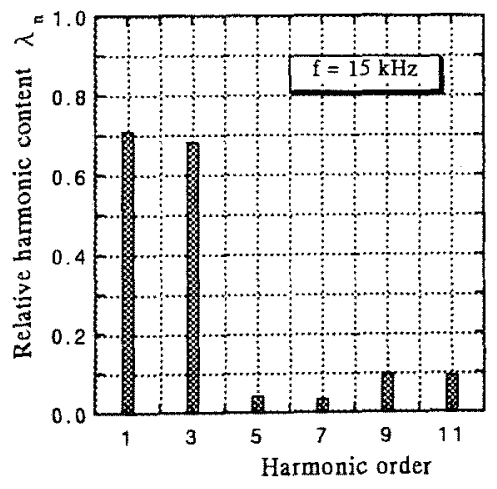

(b) $f=15 \mathrm{kHz}$

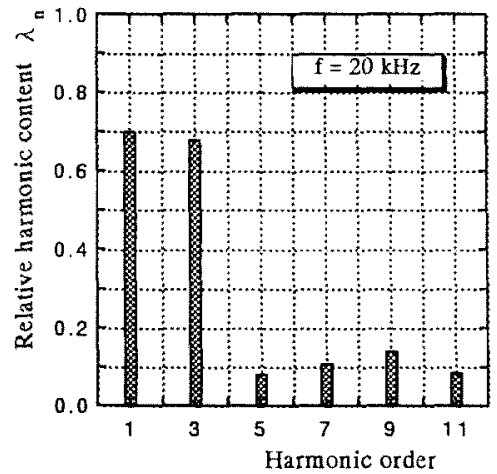

(c) $f=20 \mathrm{kHz}$

Fig.7 Experimental results of the harmonics control. 
has higher harmonics whose order are greater than 5, each content is a little. The higher order power is negligible even though higher order current is contained because the power is proportion to the square of the current. Thus there is not a serious problem.

\section{Conclusions}

A novel dual frequency output quasi-resonant inverter which positively generates higher harmonic currents has been proposed. The present inverter has a feature that the fundamental current and the 3 rd order current are controlled by manipulating the shorting time $T_{o}$ of the second resonant capacitor $C_{2}$. The absolute value of the fundamental current $I_{1}$ and the absolute value of the 3 rd order current $I_{3}$ are made nearly equal by the control. More over the fundamental frequency is adjustable. According to this inverter, simultaneous dual frequency induction heating can be possible.

In the future, how to determine the optimum value of the resonant capacitance $C_{1}$ and $C_{2}$ should be discussed in order to decrease the higher harmonic current of more than 5th order in every operation frequency.

(Manuscript received Mar. 22, 2000, revised Sept. 11, 2000)

\section{References}

[1] Yoichi Matsubara, Makoto Kumakawa and Yasuo Watanabe, "Induction Hardening of Gear by the Dual Frequency Induction Heating ", Transactions of Heat Treatment, Vol.29, No.2,1989 (in Japanese).

[2] J. Ying and K. Heumann, “ Series Resonant Inverter with Cycle Control for Induction Heating ", Proceeding of IPEMC, Vol.1, pp.367-373, 1994.

[3] E. J. Dede, V. Esteve, J. Garia, A. E. Navarro and J. A. Carrasco, " On the Design of High Frequency Series Resonant Converter for Induction Heating Applications ", Proceeding IECON, Vol.2, pp.1303-1307, 1993.

[4] S. Okudaira, K. Nomura and K. Matsuse : "New QuasiResonant Inverter for Induction Heating ”, in Conference Record of PCC-Yokohama, pp.117-122, 1993.

[5] S. Okudaira and K. Matsuse: " Adjustable Frequency Quasi-Resonant Inverter and Basic Characteristics ", Transactions of IEEJ, Vol. D-114, 1994 (in Japanese).
[6] S. Okudaira and K. Matsuse : "Frequency and Power Control of Induction Heater with Adjustable Frequency Quasi-Resonant Inverter ", Transactions of IEEJ, Vol. D118, 1997 (in Japanese).

Shizumasa Okudaira (Member) received the M.E. degree in

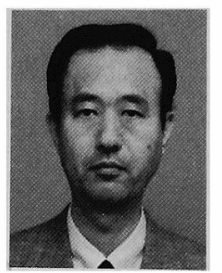
electrical engineering from Utsunomiya University, Japan, in 1980. After graduating, he entered Hitachi Ltd., and joined Tokyo Metropolitan College of Aeronautical Engineering in 1986 . He is presently an Associate Professor.

Kouki Matsuse (Member) received the B.E., M.E. and Ph.D.

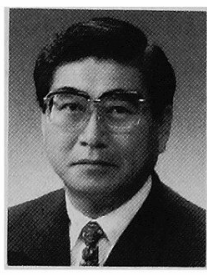
degrees in electrical engineering from Meiji University, Tokyo, Japan, in 1966, 1968 and 1971, respectively.

In 1971, he joined the faculty of Meiji University as a Lecturer of Electrical Engineering. Since 1979, he has been a Professor in the Department of Electrical Engineering and, since 1996, he has served as the Dean of the School of Science and Technology. In 1980, he was a Visiting Professor of Electrical Engineering at Iowa State University of Science and Technology, Ames, for five months. Since 1998, he has been invited to be a Guest Professor of Tsinghua University in Beijing, China. He has authored 52 published IEEE TRANSACTIONS class papers, 100 refereed full-conference papers, and is the holder of three U.S. patents. He is the coauthor of four books and coeditor of Sensorless Control of AC Motor Drives (Piscataway, NJ: IEEE Press, 1996). His research interests are power electronics, microprocessor-based controllers for static power converters and drives, adjustable-speed ac drives, and ac machines. He serves as the Chairperson of the Japanese National Committee of IEC-TC22 (Power Electronic Systems and Equipment).

Dr. Matsuse was a Member-at-Large of the IEEE Industry Applications Society (IAS) Executive Board for 1998 to 2001. He received the Outstanding Paper Award in 1992 from the IEEJ. He received the IEEE IAS Outstanding Achievement Award in 2000. He is an IEEE Fellow from 1996. 